\title{
COMPUTATIONAL ICE-DIVIDE ANALYSIS OF A COLD PLANE ICE SHEET UNDER STEADY CONDITIONS
}

\author{
by \\ F. Szidarovszky, \\ (Institute of Mathematics and Computer Science, Karl Marx University of Economics, \\ IX Dimitrov ter 8, H-1093 Budapest, Hungary) \\ K. Hutter, \\ (Institut für Mechanik, Technische Hochschule Darmstadt, Hochschulstrasse 1, \\ D-6100 Darmstadt, Federal Republic of Germany) \\ and \\ S. Yakowitz
}

(Systems and Industrial Engineering Department, University of Arizona, Tucson, AZ 85721, U.S.A.)

\begin{abstract}
The dimensionless form of the field equations and boundary conditions governing plane flow of a grounded cold ice sheet emerge from balance statements of mass, momentum, and energy. They constitute an amended version of a reduced model of ice-sheet flow, due to Morland (1984) and Hutter (1983), and circumvent the restrictions imposed by the reduced model, namely the neglect of the longitudinal stretching effects. The amended version permits satisfaction of mass balance at the ice divide for arbitrary basal sliding conditions and gives a better reproduction of the local flow features.

Under very mild simplifying assumptions, namely that horizontal thermal conduction can be ignored close to the divide, we present a numerical analysis of the ice divide which has second-order accuracy. This analysis permits determination of the temperature profile, velocity, and stress distributions in a symmetric ice divide, provided that the ice-divide height, the local behavior of the accumulation and surface-temperature functions, and the geothermal heat flow are prescribed.
\end{abstract}

\section{INTRODUCTION}

Ice divides are areas where important surrogate information about the climate or glacier history may be obtained. For example, they are the best areas for obtaining cores for isotope analysis (Raymond, 1983), and their flow regime can have a profound influence on erratic trajectories (Boulton and others, 1985), especially if one includes saddle points under the class of divides. However, ice divides present certain mathematical difficulties which (i) prevent the reduced model of Morland (1984) and Hutter (1983) from describing their evolution and characteristics, and (ii) require high-order precision in their numerical analysis when reliable local solutions of the stress, velocity, and temperature distributions are being sought.

Steady-state conditions of the reduced model were numerically studied by Yakowitz and others (1986) and Hutter and others (1986, 1987). These solutions were constructed with the aid of a marching procedure by starting at the divide, selecting a divide height, performing an ice-divide analysis, and proceeding column by column up to the margin (at which the height must vanish) was reached. The pre-selected ice-divide height was varied until the integrated accumulation function vanished for the solution to satisfy total mass balance. But, unfortunately, a large amount of basal slip was required for the predictions to be accurate. The reason for the inadequacy of the model was the neglect of longitudinal stretching effects, which we know to be significant in the vicinity of ice divides (Raymond, 1983).

Our new improved model equations incorporate the longitudinal stretching effects. They are derived from the exact continuum equations by ignoring the horizontal thermal diffusion in the heat equation (this term is known to be small). With this model, strong basal friction is now permissible, but a finite-difference solution scheme must possess at least second-order accuracy to admit an acceptable local solution. Even higher-order accuracy is needed if a complete ice-sheet solution is to be found.

We will demonstrate this analysis for plane-flow conditions and a locally symmetric ice divide. The analysis of a dome of a locally axisymmetric ice sheet is analogous and only requires minor changes. Basic equations will only be briefly stated and not derived, as they can be found, for instance, in Hutter (1983).

\section{GOVERNING EQUATIONS}

The equations of the model prior to any approximations are as follows.

\subsection{Ficld equations}

When restricted to plane flow, the dimensionless velocities $U, W$, stresses $\sigma_{x}, \sigma_{z}, \tau$, and the temperature $T$ are governed by the equations (see Fig. 1):

$$
\begin{aligned}
& \text { (a) } \varepsilon \frac{\partial \sigma_{x}}{\partial X}+\frac{\partial \tau}{\partial Z}=0, \\
& \text { (b) } \varepsilon \frac{\partial \tau}{\partial X}+\frac{\partial \sigma_{z}}{\partial Z}=1, \\
& \text { (c) } \frac{\partial U}{\partial X}+\frac{\partial W}{\partial Z}=0, \\
& \text { (d) } \frac{\partial U}{\partial X}=\frac{1}{v} a(T) \omega\left(\tau_{\mathrm{II}} / s^{2}\right) \frac{1}{2}\left(\sigma_{x}-\sigma_{z}\right),
\end{aligned}
$$




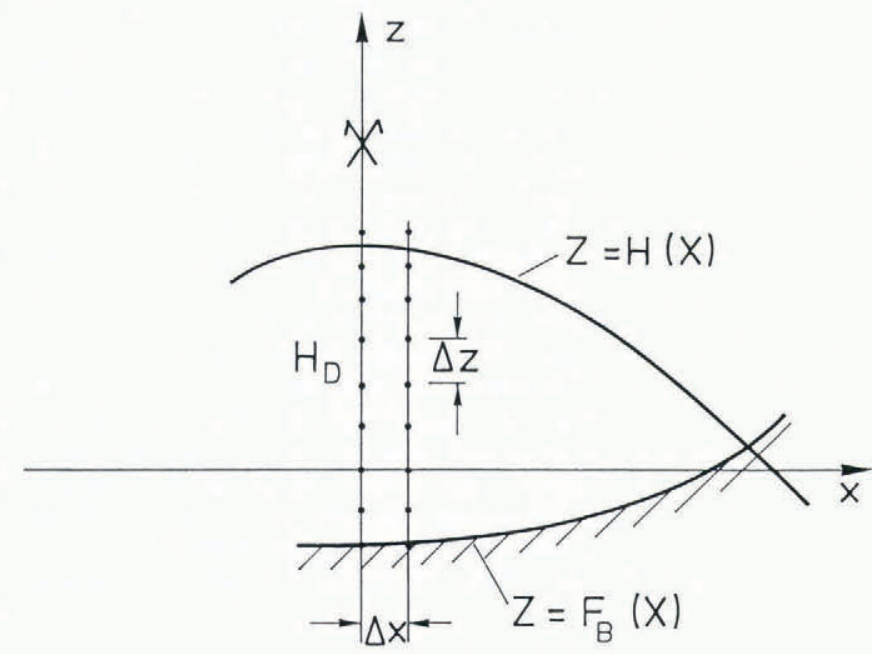

Fig. 1. Symmetric plane ice sheet indicating the coordinates, the free surface, and the bottom profiles, including a sketch of the finite-difference approximation close to the divide.

TABLE Ia. SCALES USED TO NON-DIMENSIONALIZE THE FIELD EQUATIONS AND BOUNDARY CONDITIONS. NUMERICAL VALUES ARE TYPICAL FOR THE SITUATION. A RANGE OF VALUES IS DISCUSSED IN THE TEXT

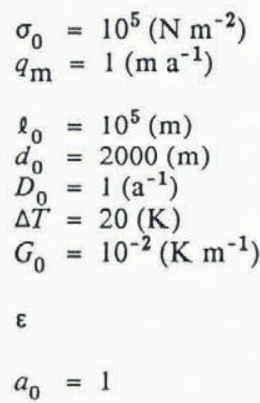

TABLE Ib. PHYSICAL PARAMETERS

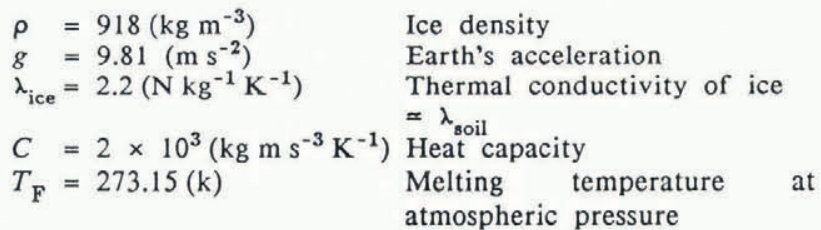

TABLE IC. DIMENSIONLESS PARAMETERS BASED ON THE ABOVE SCALES

$s=\frac{\sigma_{0}}{\rho g d_{0}}=10^{-4}-10^{-1}$,

$$
\alpha=\frac{g d_{0}}{C \Delta T}=10^{-2}-10^{0},
$$

$\psi=\frac{q_{\mathrm{m}}}{d_{0} D_{0} a_{0}}=10^{-4}-10^{-1}$,

$$
\beta=\frac{\lambda_{\text {ice }}}{\rho C d_{0} q_{\mathrm{m}}}=10^{-2}-10^{0},
$$

$v=\delta \cdot s$,

$$
\varepsilon=d_{0} / \ell_{0}=10^{-3}-10^{-1},
$$

$\gamma=\frac{\rho g d_{0}^{2} q_{\mathrm{m}}}{\Delta T \lambda_{\text {ice }} \varepsilon}=10^{0}-10^{2}$, $\xi=\frac{\lambda_{\text {soil }}}{\lambda_{\text {ice }}} \frac{G_{0} d_{0}}{\Delta T} \simeq 10^{-1}-10^{0}$.

(In the reduced model equations $v=\varepsilon^{2}$ and $\theta=\psi / s$.)
TABLE IIa. MATERIAL RESPONSE FUNCTIONS FOR POLYCRYSTALLINE ICE

$$
\begin{aligned}
a(T) & =a_{0} \exp \left(b_{0} \cdot T\right)+a_{1} \exp \left(b_{1} \cdot T\right) \\
\omega(X) & =c_{0}+c_{1} X+c_{2} X^{2}, \text { where } X=\tau_{\mathrm{II}} \\
a_{0} & =0.7242, b_{0}=11.9567, a_{1}=0.3438, b_{1}=2.9494 \\
c_{0} & =0.3336, c_{1}=0.3200, c_{2}=0.02963
\end{aligned}
$$

TABLE IIb. ACCUMULATION-RATE AND SURFACETEMPERATURE FUNCTIONS (EXAMPLES)

$$
\begin{aligned}
& A_{\mathrm{cc}}(X, H(X))=A_{0}\left[1-\exp \left(-\left(H-H_{\mathrm{e}}(X)\right) / A_{1}\right]\right. \\
& T_{\mathrm{S}}(X, H(X))=T_{0}-T_{1} H(X)-T_{2} X \\
& H_{\mathrm{e}}(X)=H_{0}\left(1-H_{2} X^{2}\right) \\
& A_{0}=0.5, \quad A_{1}=0.2-0.05 \\
& T_{0}=-0.1, \quad T_{1}=0.5-1.0, \quad T_{2}=0-10^{-1} \\
& H_{0}=0.5-1.5, H_{2}=10^{-2}-10^{-1}
\end{aligned}
$$

TABLE IIC. BASAL SLIDING LAW AND GEOTHERMAL HEAT FLOW

$$
\begin{aligned}
Q^{\text {geoth }}\left(X, F_{\mathrm{B}}(X)\right) & =1, \\
U_{\mathrm{F}}\left(\left|\sigma_{z}\right|, \tau, T\right) & =\frac{1}{\left|\sigma_{z}\right|} \frac{1}{\mu} \tau, \mu \sim 10
\end{aligned}
$$

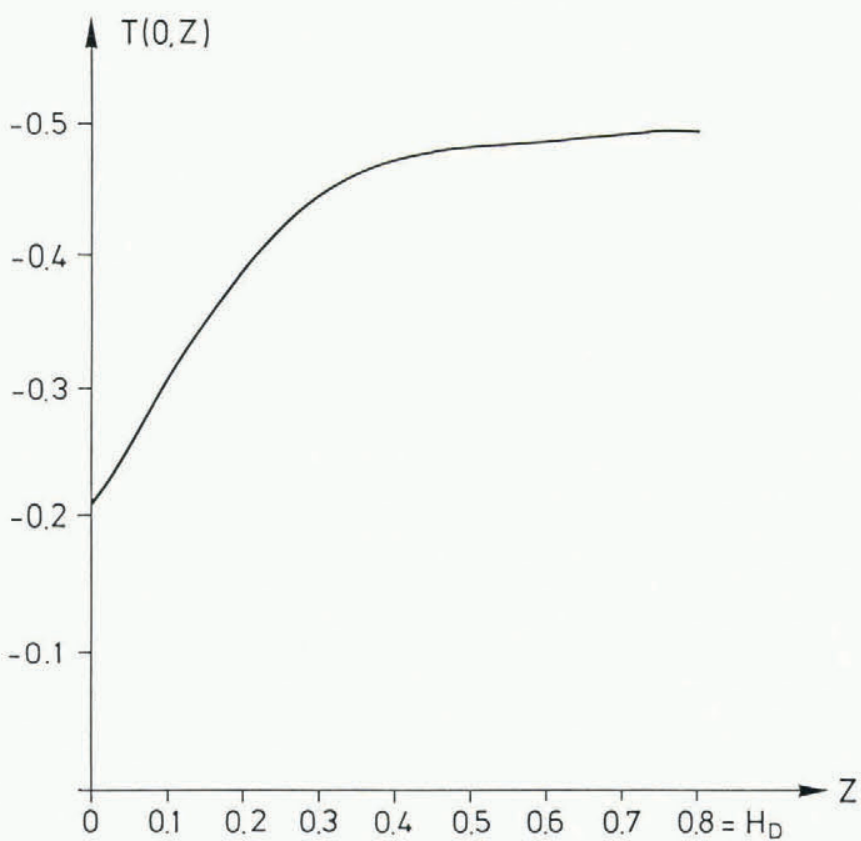

Fig. 2. Typical shape of the temperature distribution at the divide $\left(Z=0\right.$ corresponding to the bottom, $Z=H_{\mathrm{D}}$ to the top). 
(e) $\frac{\partial U}{\partial Z}+\varepsilon^{2} \frac{\partial W}{\partial X}=\frac{2 \varepsilon}{v} a(T) \omega\left(\tau_{\mathrm{II}} / s^{2}\right) \tau$,

(f) $\frac{\partial T}{\partial t}+U \frac{\partial T}{\partial X}+W \frac{\partial T}{\partial Z}=$

$$
=\beta\left[\frac{\partial^{2} T}{\partial Z^{2}}+\varepsilon^{2} \frac{\partial^{2} T}{\partial X^{2}}\right]+\frac{2 \alpha}{v} a(T) \omega\left(\tau_{\mathrm{II}} / s^{2}\right) \tau_{\mathrm{II}}
$$

where $a(T)$ is a temperature-dependent rate function, $\omega\left(\tau_{\mathrm{II}} / s^{2}\right)$ is a creep response function, and

$$
\tau_{\mathrm{II}}=\frac{1}{4}\left(\sigma_{x}-\sigma_{z}\right)^{2}+\tau^{2}=\sigma_{x}^{\prime 2}+\tau^{2}
$$

is the second stress-deviator invariant (see Hutter and others, 1986). $s, v$, and $\varepsilon$ are dimensionless constants, which are independent here but were given by $s=\varepsilon \theta^{-1 / 2}$ and $v=\varepsilon^{2}$ in the reduced model. We still may assume that $\varepsilon$ and $v$ are smaller than unity and that $\theta$ is $O(1)$ or larger, but an inter-relationship is not presently suggested. In addition, $\alpha$ and $\beta$ are given constants; they are called an energy-dissipation and a thermal diffusion number, respectively. In terms of physical constants and typical scales, these constants are given in Table I. Numerical values have a considerable spread.

In Equations (1), statements (a) and (b) are a horizontal force balance, (c) is the incompressibility condition, while (d) and (e) are constitutive relations of stress relating strain-rate in a non-linear fashion to the stress deviator. The non-linearity is expressed by the temperature-dependent rate factor $a(T)$ and the creep response function $\omega\left(\tau_{\mathrm{II}} / s^{2}\right)$. Finally, (f) is the heat equation that incorporates advection, thermal diffusion, and strain heating. As shown by Morland (1984), all these terms are significant in thermo-mechanical coupled ice-sheet dynamics.

Rate factors $a(T)$ between 263 and $273 \mathrm{~K}$ satisfy a polynomial relation $a(T)=P_{3}(T)$. Likewise, $\omega\left(\tau_{\mathrm{II}} / s^{2}\right)$ is of ten a power law, but numerous laboratory and field studies (see Hutter, 1983, chapter 2) have indicated that polynomial expressions fit available data better. We shall adopt a "finite viscosity" expression to avoid mathematical singularities at the free surface (Johnson and McMeeking, 1984) but leave both $a(T)$ and $\omega\left(\tau_{\mathrm{II}} / s^{2}\right)$ arbitrary to adjust for local constitutive behavior. A choice is given in Table II.

The constitutive relations of Equations ( $1 \mathrm{~d}$ and e) do not incorporate any stress-induced anisotropies (i.e. directional dependencies). To our knowledge, such anisotropy effects have so far not been incorporated in any glaciological context. However, inhomogeneities (i.e. positional dependencies) can easily be incorporated in the rate factors as well as the creep-response function by making $a$ and $w$ also position-dependent. So, our formulation permits incorporation of the differences of the softening effect of the dust particles in Pleistocene ice by an internal variable (see Hutter and Vulliet, 1985) or simply by making $a$ and $\omega$ position-dependent.

\subsection{Boundary conditions}

The field Equations (1) and (2) are complemented by boundary conditions at the free surface

$$
\begin{aligned}
\frac{\partial H}{\partial t}+U \frac{\partial H}{\partial X}-W & =A_{\mathrm{cc}}(X, H, t), \\
-\sigma_{X} \varepsilon \frac{\partial H}{\partial X}+\tau & =p_{\mathrm{atm}} \varepsilon \frac{\partial H}{\partial X},
\end{aligned}
$$

$$
\begin{gathered}
-\tau \varepsilon \frac{\partial H}{\partial X}+\sigma_{z}=-p_{\mathrm{atm}}, \\
T=T_{\mathrm{S}}(X, H, t),
\end{gathered}
$$

and at the base

$$
\begin{aligned}
& \text { (e) } U=U_{\mathrm{F}}\left[\left|\sigma_{z}\right|, \tau, T\right] \text {, } \\
& \text { (f) } W=U \frac{\mathrm{d} F_{\mathrm{B}}}{\mathrm{d} X} \text {, } \\
& \text { (g) } \varepsilon^{2} \frac{\mathrm{d} F_{\mathrm{B}}}{\mathrm{d} X} \frac{\partial T}{\partial X}-\frac{\partial T}{\partial Z}=+\gamma\left[\tau-\varepsilon\left(\sigma_{x}-\sigma_{z}\right) \frac{\mathrm{d} F_{\mathrm{B}}}{\mathrm{d} X}-\right. \\
& \text { at } Z=F_{\mathrm{B}}(X) \\
& \left.-\varepsilon^{2} \tau\left[\frac{\mathrm{d} F_{\mathrm{B}}}{\mathrm{d} X}\right)^{2}\right] U+ \\
& +\zeta_{\perp}^{\text {geoth }}\left(X, F_{\mathrm{B}}(X)\right)\left[1+\varepsilon^{2}\left[\frac{\partial F_{\mathrm{B}}}{\partial X}\right]^{2}\right]^{\frac{1}{2}} .
\end{aligned}
$$

In Equations (3), (a) is a kinematic statement expressing the evolution of the surface profile; (b) and (c) are surface-stress conditions relating $\sigma_{x}, \sigma_{z}$, and $\tau$ to the atmospheric pressure but ignoring the small wind-stress term. Relation (d) is the Dirichlet condition of temperature, i.e. we prescribe the surface temperature through $T_{S}(X, H, t)$. At the base, a sliding condition is applied. This is formally implemented in Equations (4) by prescribing the horizontal velocity component (Equation (e)) and requesting tangency of the sliding velocity along the base $Z=F_{\mathrm{B}}(X)$, expressed in Equation ( $f$ ). Statement $(g)$ equates the heat flow from the basal surface into the ice to the dimensionless frictional heat plus the dimensionless geothermal heat flow $Q I^{\text {geoth }}\left(X, F_{\mathrm{B}}, t\right)$ which is positive as a flow towards the ice. Orders of magnitude for $Q_{1}^{\text {geoth }}$ are $0.1-1.0$. The term involving $\gamma$ represents generation of frictional heat along the base; $\gamma$ is a dimensionless quantity with the order of magnitude $\leqslant 10^{2}$; it is also defined in Table $\mathrm{I}$.

We emphasize that the boundary condition in Equations
(3)-(4) are exact statements valid for any value of $\varepsilon$. The climatology input is provided by $p_{\text {atm }}$ (usually $=0$ ) and the two forcing functions $A_{\mathrm{c}}($.$) and T_{\mathrm{S}}($.$) , and the interaction$ with the substratum is described by the geothermal heat flow $Q_{\perp}^{\text {geoth }}($.$) and the sliding law U_{\mathrm{F}}($.$) .$

The accumulation rate has generally a strong dependence on $H$. Denoting by $H_{\mathrm{E}}$ the snow-equilibrium height, we have $A_{\mathrm{cc}}>0$ if $H>H_{\mathrm{E}}$ and $A_{\mathrm{cc}}<0$ if $H<H_{\mathrm{E}}$ with $A_{\mathrm{cc}}=0$ at $H=H_{\mathrm{E}}$. A polynomial dependence on the variable $\left(H-H_{\mathrm{E}}\right)$ may be appropriate, but an $X$ - or $t$-dependence can also be incorporated by choosing $H_{\mathrm{E}}=H_{\mathrm{E}}(X, t)$.

The surface temperature $T_{\mathrm{S}}$ is the temperature measured $10 \mathrm{~m}$ below the surface. It also has a strong dependence on height, which in a first approximation can be assumed to be linear.

The geothermal heat flow $Q_{\perp}^{\text {geoth }}$ is of ten assumed to be constant (corresponding to $3^{\circ} \mathrm{C} / 100 \mathrm{~m}$ ). However, it may equally be assumed to be a function of position and time 
Computationally, it is also advantageous to prescribe the geothermal heat through a "virtual" horizontal plane,

$$
Q^{\text {geoth }}\left(X, F_{\mathrm{B}}(X)\right)=Q^{\text {geoth }}\left(X, F_{\mathrm{B}}(X)\right)\left[1+\varepsilon^{2}\left[\frac{\partial F_{\mathrm{B}}}{\partial X}\right)^{2}\right]^{\frac{1}{2}} \text {, }
$$

rather than $Q_{\perp}^{\text {geoth }}$. Values of $Q^{\text {geoth }}\left(X, F_{\mathrm{B}}(X)\right)$ are generally larger in the central region than towards the margin. This follows simply from the insulating effect that is exhibited by the ice sheet.

The sliding law is probably the most complicated of all. It must be selected with caution because both physical and mathematical arguments will determine its form (Hutter, 1983). For a finite slope margin (wedge type), one must have $U_{\mathrm{F}}=c_{2}\left|\sigma_{z}\right|^{\tau}$ as the margin position is approached. In other words, the sliding velocity is linear in the shear traction and the overburden pressure.

The explicit forms of all these functional relations used in this paper are given in Table II.

We now give a heuristic proof that Equations (1) can circumvent the restriction that basal sliding must be large. To this end, recall that, from Hutter and others (1986), the curvature of the surface profile at the ice divide, computed according to the reduced equations is given by

$$
H_{\mathrm{D}}^{\prime \prime}=\frac{-A_{\mathrm{cc}}\left(0, H_{\mathrm{D}}\right)}{\text { Glide }+ \text { Slide }}
$$

where

$$
\begin{aligned}
& \text { Slide }=\left[\varepsilon^{2}\left(H_{\mathrm{D}}-F_{\mathrm{B}}\right)\right] /\left[\mu_{\operatorname{Ref}} \tilde{\mu}\left(H_{\mathrm{D}}-F_{\mathrm{B}}\right)\right], \\
& \text { Glide }=\int_{F_{\mathrm{B}}}^{H_{\mathrm{D}}} 2 \omega\left(\tau_{\mathrm{II}} / s^{2}\right)\left(H_{\mathrm{D}}-F_{\mathrm{B}}\right)^{2} a(T(0, z)) \mathrm{d} z,
\end{aligned}
$$

and $H_{\mathrm{D}}=H(0), \quad F_{\mathrm{B}}=F_{\mathrm{B}}(0), \quad \sigma_{\mathrm{X}}(0)=\sigma_{\mathrm{Z}}(0), \quad$ and thus, $\tau_{\mathrm{II}}(0)=0$ since $\tau(0)=0$. With $\tau_{\mathrm{II}}=0$ at the divide, $\omega=$ $\omega(0)$ which vanishes for a power flow law implying GLIDE = 0 . This necessarily requires SLIDE $\neq 0$. We have chosen $\omega(0) \neq 0$ and thus would make $H^{\prime \prime}$ dependent upon a "finite viscosity at zero stress", a quantity which is poorly known. In addition, GLIDE is very small which again requires that SLIDE is of order unity. The point is that the shallow-ice approximation yields doubtful results close to the divide. For the improved model, longitudinal stretching is significant so $\sigma_{\mathrm{X}}(0) \neq \sigma_{\mathrm{Z}}(0)$ cannot vanish even with $\tau(0)=$ 0 .

Balancing both sides of Equation (1d) then requires $\sigma_{x}^{\prime}=\frac{1}{2}\left(\sigma_{x}-\sigma_{z}\right)=v \bar{\sigma}_{x}^{\prime}$ so that Equations (1c and d) with $\tau=\varepsilon \bar{\tau}$ reduce to

$$
\begin{aligned}
& \frac{\partial U}{\partial X}+\frac{\partial W}{\partial Z}=0, \\
& \frac{\partial U}{\partial X}=a(T) \omega\left(\tau_{\mathrm{II}} / s^{2}\right) \bar{\sigma}_{X}^{\prime} .
\end{aligned}
$$

At a symmetric ice divide, Equations (1a) and (le) are identically satisfied, and we may write in accordance with mass balance

$$
W \simeq-A_{\mathrm{cc}}\left[\frac{Z-F_{\mathrm{B}}}{H_{\mathrm{D}}-F_{\mathrm{B}}}\right],
$$

and then deduce from above

$$
\frac{\partial U}{\partial X}=\frac{A_{c c}}{H_{\mathrm{D}}-F_{\mathrm{B}}}=a(T) \omega\left(\tau_{\mathrm{II}} / s^{2}\right) \bar{\sigma}_{x}^{\prime} .
$$

This relation defines both $\partial U / \partial X$ and $\sigma_{x}^{\prime}$ at the divide, if $T$ and $H$ are prescribed. With $\bar{\sigma}_{x}^{\prime} \neq 0$, one has $\tau_{\mathrm{II}} \neq 0$, $\omega\left(\tau_{\mathrm{II}}\right) \neq 0$, and thus GLIDE $\neq 0$. In fact, we shall demonstrate that $\omega \leqslant 0(1)$ under usual circumstances and so SLIDE may vanish without making $H_{\mathrm{D}}^{\prime \prime}$ infinite.

Henceforth, steady-state conditions will be considered and time derivatives in Equations (1f) and (3a) will be omitted.

\subsection{Numerical solution strategy}

Suppose we ignore the terms $\varepsilon^{2} \partial^{2} T / \partial X^{2}$ in the energy Equation (If). The soundness of this approximation can be tested a posteriori. Let the finite-difference approximation of the derivative of $f$ with respect to $X$ be denoted by $\delta f$. (We shall define $\delta f$ below.) Then, Equations (1) and (2) may be written as

(a) $\frac{\partial \tau}{\partial Z}=-\varepsilon \delta \sigma_{x}$,

(b) $\frac{\partial \sigma_{z}}{\partial Z}=1-\varepsilon \delta \tau$,

(c) $\frac{\partial W}{\partial Z}=-\delta U$,

(d) $\frac{\partial U}{\partial Z}=-\varepsilon^{2} \delta W+\frac{2 \varepsilon}{v} a(T) \omega\left(\tau_{\mathrm{II}} / s^{2}\right) \tau$,

(e) $\frac{\partial T}{\partial Z}=T^{\prime}$,

(f) $\frac{\partial T^{\prime}}{\partial Z}=\frac{1}{\beta}\left[U \delta T+W T^{\prime}-\frac{2 \alpha}{v} a(T) \omega\left(\tau_{\mathrm{II}} / s^{2}\right) \tau_{\mathrm{II}}\right]$,

with

$$
\sigma_{x}=\sigma_{z}+\frac{2 v \delta U}{a(T) \omega\left(\tau_{\mathrm{II}} / s^{2}\right)},
$$

$$
\tau_{\mathrm{II}}=\left[\frac{\mathrm{v} \delta U}{a(T) \omega\left(t_{\mathrm{II}} / s^{2}\right)}\right]^{2}+\tau^{2} .
$$

Note that Equation (7a) is a re-arrangement of Equation (Id) and Equation (7b) is a combination of Equation (7a) and the definition of $\tau_{\mathrm{II}}$, in Equations (2).

The boundary conditions are as follows:

at $Z=F_{\mathrm{B}}(X)$ :

(a) $W=U \delta F_{\mathrm{B}}$,

(b) $T^{\prime}=-\xi Q^{\text {geoth }}(X)+\varepsilon^{2} \delta T \delta F_{\mathrm{B}}-$

$$
\text { - } \gamma U\left[\tau-e\left(\sigma_{x}-\sigma_{z}\right) \delta F_{\mathrm{B}}-\varepsilon^{2} \tau\left(\delta F_{\mathrm{B}}\right)^{2}\right],
$$

(c) $U=U_{\mathrm{F}}\left(\left|\sigma_{z}\right|, \tau, T\right)$,

and at $Z=H(X)$ :

(a) $U \cdot H^{\prime}-W=A_{\mathrm{cc}}(X, H(X))$,

(b) $-\sigma_{x} \varepsilon H^{\prime}+\tau=\varepsilon H^{\prime} p_{\text {atm }}$

(c) $-\tau \varepsilon H^{\prime}+\sigma_{z}=-p_{\mathrm{atm}}$,

(d) $\quad T=T_{\mathrm{S}}(X, H(X)$.

Observe that for any fixed value of $x$ Equations (7) and boundary conditions (9) and (10) define a two-point boundary value problem (TPBVP) for the unknown functions $\tau, \sigma_{z}, W, U, T$, and $T^{\prime}$, provided that the approximating derivatives $\delta \sigma_{x}, \delta \tau, \delta U, \delta W, \delta T$, and $\delta F_{\mathrm{B}}$ 
are known.

In order to take advantage of this property, the solution strategy is as follows. For any given value of the divide height $H_{\mathrm{D}}$, we shall proceed in three steps:

Step 1. All unknowns will be determined at the divide and in the column next to the divide. (That is, for $x=0$ and for $x=\Delta x$.)

Step 2. Using a marching procedure, the unknown functions will be determined for all $x=k \cdot \Delta x(k \geqslant 2)$, such that $H(k \Delta x)>0$. The margin position $x_{\mathrm{M}}\left(H_{\mathrm{D}}\right)$ is reached when for the first time $H((k+1) \Delta x) \leqslant 0$.

Step 3. The divide height $H_{\mathrm{D}}$ is determined by the condition that the integrated squared accumulation must vanish. That is, the value of $H_{\mathrm{D}}$ is obtained by solving the equation ( $T_{\mathrm{a}}$ is called the target function)

$$
T_{\mathrm{a}}\left(H_{\mathrm{D}}\right)^{\mathrm{DEF}}\left[\int_{0}^{X} A_{\mathrm{cc}}(X, H(X)) \mathrm{d} x\right]^{2}=0 .
$$

For example, the secant method (Yakowitz and Szidarovszky, 1986) can be used for the numerical solution of this equation. The advantage of using the secant method is the fact that this method does not use derivatives of the target function. Note that the computation of the target function at any value of $H_{\mathrm{D}}$ requires the completion of steps 1 and 2 for this fixed value of $H_{\mathrm{D}}$

In this paper, we shall not discuss the marching process in general; we shall leave this step to a succeeding paper. But step 1 will be discussed in detail.

We now demonstrate how the ice-divide analysis is performed.

\section{NUMERICAL TREATMENT OF THE ICE DIVIDE}

For simplicity, a symmetric configuration will be considered.

\subsection{Divide analysis}

At a symmetric ice divide the following symmetry conditions must be fulfilled:

$$
\begin{array}{ll}
U(0, Z)=0, & \frac{\partial T}{\partial X}(0, Z)=0, \\
\frac{\partial W}{\partial X}(0, Z)=0, & \tau(0, Z)=0, \\
\frac{\partial \sigma_{x}}{\partial X}(0, Z)=\frac{\partial \sigma_{z}}{\partial X}(0, Z)=0, & H^{\prime}(0)=0 .
\end{array}
$$

Substituting these relations into the steady-state versions of the governing field Equations (1) and boundary conditions (2) and (3), the following system of equations
(a) $\frac{\partial \sigma_{z}}{\partial Z}=1-\varepsilon \frac{\partial \tau}{\partial X}$,
(b) $W(Z)=W^{(0)}(Z)$,
(c) $\frac{\partial T^{\prime}}{\partial Z}=\frac{1}{\beta}\left[W T^{\prime}-\frac{2 \alpha}{v} a(T) \omega\left(\tau_{\mathrm{II}} / s^{2}\right) \tau_{\mathrm{II}}\right]$,
(d) $\frac{\partial T}{\partial Z}=T^{\prime}$,
(e) $\frac{\partial W}{\partial Z}=-\frac{1}{v} a(T) \omega\left(\tau_{\mathrm{II}} / s^{2}\right) \frac{\Delta}{2}$

with boundary conditions

$$
\left.\begin{array}{l}
\text { (a) } T^{\prime}=-\xi Q^{\text {geoth }}(0), \text { at } Z=F_{\mathrm{B}}(0), \\
\text { (b) } T=T_{\mathrm{S}}[0, H(0)], \\
\text { (c) } \sigma_{z}=-P_{\text {atm }}(0),
\end{array}\right\} \text { at } Z=H(0)
$$

is obtained. In the above

$$
\Delta=\sigma_{\mathrm{X}}-\sigma_{\mathrm{Z}}\left(=\frac{1}{2} \sigma_{\mathrm{X}}^{\prime}\right), \tau_{\mathrm{II}}=\frac{\Delta^{2}}{4}
$$

and $W^{(0)}(Z)$ is a prescribed function. Our initial - and later improved - ice-divide analysis will therefore be based on an approximate representation of the vertical velocity profile, the first estimate being based upon the linear relation

$$
W^{(0)}(Z)=-A_{\mathrm{cc}}[0, H(0)] \frac{Z-F_{\mathrm{B}}(0)}{H(0)-F_{\mathrm{B}}(0)} .
$$

Note also that, since $\omega$ is increasing in $\Delta^{2}$ and $\Delta$ is a multiplier, there is a unique solution $\Delta$ of Equation (13e) for any values of $H(0)$ and $T$. For the suggested numerical procedure this is important. The procedure is now as follows:

Equations (13c, d, and e) with the definitions in Equation (15) and the boundary conditions in Equations (14a and b) yield a TPBVP for $T^{\prime}$ and $T$. In the solution of this TPBVP, $\Delta$ is obtained by solving the non-linear Equation (13e) using thereby the estimate in Equation (16); $\tau_{\text {II }}$ then follows from Equation $(15 \mathrm{a}, \mathrm{b})$. Finally, we may recall that $U=\tau=0$ at the divide.

Note that the functions $\sigma_{\mathrm{X}}$ and $\sigma_{\mathrm{Z}}$ are not determined; only their difference, function $\Delta$, is obtained. As we shall see later, in the column next to the divide a simultaneous computation of all variables with those at the divide is possible which makes all the variables available.

\subsection{Second-order derivative approximation}

We list here without proof a first $X$-derivative approximation of second order, i.e. the error is $0\left(\Delta X^{2}\right)$. Let $X=0$ be the point of symmetry. Consider the derivative $\partial f / \partial X$. Then to second order,

$$
\frac{\partial f}{\partial X}=\delta f+0\left(\Delta X^{2}\right)
$$

where

$$
\delta f(X)=\left\{\begin{array}{l}
0, \text { if } x=0 \text { and } f \text { is even, } \\
\frac{2(f(X)-f(0))}{\Delta X}, \text { if } X=\Delta X \text { and } f \text { is even, (b) } \\
\frac{f(X)}{\Delta X}, \text { if }(X=0 \text { or } X=\Delta X) \text { and } f \text { is odd, (c) } \\
\frac{3 \mathrm{f}(X)-4 f(X-\Delta X)+f(X-2 \Delta X)}{2 \Delta X}, \text { if } X>\Delta X .
\end{array}\right.
$$

This formula will be used repeatedly in what follows.

We also need the backward second derivative in $Z$

$$
\frac{\partial^{2} f}{\partial Z^{2}}(0, Z)=\delta^{2} f(0, Z)+0\left(\Delta Z^{2}\right)
$$

where

$$
\begin{aligned}
\delta^{2} f(0, Z)= & \frac{1}{\Delta Z^{2}}[2 W(0, Z)-5 W(0, Z-\Delta Z)+ \\
& +4 W(0, Z-2 \Delta Z)-W(0, Z-3 \Delta Z)]
\end{aligned}
$$


In the above, even functions satisfy the property $f(X)=$ $f(-X)$ while for odd functions one has $f(X)=-f(-X)$.

\subsection{Column next to the divide}

Recall that at the divide we know the functions $W, T$, $T^{\prime}, \Delta$, and $U=\tau=0$.

Assume now that $X=\Delta X$. Then, up to second order, the even functions are the same at the divide and in the column next to the divide. Thus
(a) $W(\Delta X, Z) \approx W(0, Z)$,
(b) $T(\Delta X, Z) \approx T(0, Z), \quad 0 \leqq z \leqq H(0)$.
(c) $\Delta(\Delta X, Z) \approx \Delta(0, Z)$.

The other functions $U, \tau, \sigma_{x}$, and $\tau_{\mathrm{II}}$ at the divide and next to the divide can be obtained simultaneously in several steps.

Step 1

From Equation (7c) and relations (17c and d), we may deduce the approximations

$$
U(\Delta X, Z)= \begin{cases}\frac{\Delta X}{2 \Delta Z}[3 W(0, Z)-4 W(0, Z+\Delta Z)+W(0, Z+2 \Delta Z)], & \text { if } Z=F_{\mathrm{B}}(0) \\ -\frac{\Delta X}{2 \Delta Z}[3 W(0, Z)-4 W(0, Z-\Delta Z)+W(0, Z-2 \Delta Z)], & \text { if } Z=H(0), \\ -\frac{\Delta X}{2 \Delta Z}[W(0, Z+\Delta Z)-W(0, Z-\Delta Z)], & \text { if } F_{\mathrm{B}}(0)<Z<H(0)\end{cases}
$$

Thus, $U(\triangle X, Z)$ is now known for all $Z$.

Step 2

By using the boundary conditions in Equations (3) at $Z=H(0)$, the values of $\sigma_{x}(\Delta X, H(0)), \quad \sigma_{z}(\Delta X, H(0))$, $\tau(\Delta X, H(0))$ can be determined with an estimated value of $H^{\prime}(\Delta X) . \quad\left(H^{\prime}(\Delta X)\right.$ evaluated from Equation (3a) would be zero if second-order accuracy is used.) Then Equations (3b and c) and the definition of $\Delta$ imply for $X=\Delta X$ and $Z=H(0)$,

$$
\begin{aligned}
\sigma_{x} & =\frac{\Delta}{1-\varepsilon^{2} H^{\prime 2}}-p_{\mathrm{atm}}, \\
\tau & =p_{\mathrm{atm}} \varepsilon H^{\prime}+\sigma_{x} \varepsilon H^{\prime}, \\
\sigma_{z} & =\sigma_{x}-\Delta,
\end{aligned}
$$

all of which can be computed.

Step 3

We iterate on Equations (21) by constructing improved values of $H^{\prime}$. From a Taylor-series expansion of $H^{\prime}$ we have

$$
H^{\prime}(\delta X)=H_{\mathrm{D}}^{\prime \prime} \Delta X+0\left(\Delta X^{2}\right) .
$$

From a Taylor-series expansion in $X$ of the steady version of Equation (3a), it follows that

$$
H_{\mathrm{D}}^{*}=-\frac{\left[\frac{\partial^{2} A_{\mathrm{cc}}\left(0, H_{\mathrm{D}}\right)}{\partial X^{2}}+\frac{\partial^{2} W\left(0, H_{\mathrm{D}}\right)}{\partial X^{2}}\right]}{\left[3 \frac{\partial W\left(0, H_{\mathrm{D}}\right)}{\partial Z}+\frac{\partial A_{\mathrm{cc}}\left(0, H_{\mathrm{D}}\right)}{\partial Z}\right]} .
$$

The derivative terms of $A_{\mathrm{cc}}($.$) can be computed to any$ desired order of numerical accuracy, because this function is prescribed. $\partial W\left(0, H_{\mathrm{D}}\right) / \partial Z$ is given by Equation (16), but $\partial^{2} W\left(0, H_{\mathrm{D}}\right) / \partial X^{2}$ is still unknown; it can be determined in the following way.

By differentiating Equation (1e) with respect to $X$ and imposing the symmetry relation $\tau(0, Z)=0$, we obtain

$$
\frac{\partial^{2} W}{\partial X^{2}}=\frac{1}{\varepsilon^{2}}\left[\frac{2 \varepsilon}{v} a(T) \omega\left(\frac{\tau_{\mathrm{II}}}{s^{2}}\right) \frac{\partial \tau}{\partial X}+\frac{\partial^{2} W}{\partial Z^{2}}\right],
$$

so that with the aid of Equations (17c) and (18) we obtain for $Z=H(0)$

$$
\begin{aligned}
\frac{\partial^{2} W(0, Z)}{\partial X^{2}}= & \frac{1}{\varepsilon^{2}}\left[\frac{2 \varepsilon}{v} a[T(0, Z)] \omega\left[\frac{\Delta^{2}}{4 s^{2}}\right] \frac{\tau(\Delta X, Z)}{\Delta X}+\right. \\
& +\frac{1}{\Delta Z^{2}}[2 W(0, Z)-5 W(0, Z-\Delta Z)+ \\
& +4 W(0, Z-2 \Delta Z)-W(0, Z-3 \Delta Z)]] .
\end{aligned}
$$

With Equation (25), an improved estimate of $H_{\mathrm{D}}^{\prime \prime}$ and therefore that of $H^{\prime}(\Delta X)$ is computed as given by Equations (22) and (23). Step 2 then provides improved values for $\sigma_{\mathrm{X}}(\Delta X, H), \sigma_{\mathrm{z}}(\Delta X, H), \tau(\Delta X, H)$.

Step 4

Similarly, at the divide we have from the boundary condition (14c)

$$
\sigma_{z}(0, H(0))=-p_{\text {atm }}
$$

and therefore, since $\Delta$ is known at the divide,

$$
\sigma_{x}(0, H(0))=\sigma_{z}(0, H(0))+\Delta(0, H(0)) .
$$

Furthermore, we know that

$$
\tau(0, H(0))=0 \text {, and also } \tau(0, Z)=0 \text { for all } Z \text {. }
$$

Step 5

Starting from the free surface, we show how a marching procedure down the two columns at the divide and next to it can be developed to obtain all these functions $\sigma_{x}(0, Z), \quad \sigma_{z}(0, Z), \quad \sigma_{x}(\Delta X, Z), \quad \sigma_{z}(\Delta X, Z)$, and $\tau(\Delta X, Z)$ for all values of $Z$. For $Z=H(0)$, they are all known. Let now $Z$ be given, and assume that all the above functions are known for $Z+\Delta Z$. Then the procedure is as follows: in Equation (7b), the respective derivative approximations of $\partial \sigma_{Z}(\Delta X, Z+\Delta Z) / \partial Z$ and $\delta \tau(\Delta X, Z+\Delta Z)$ are substituted. Together with Equation (19c) this yields 


$$
1-\varepsilon \frac{\tau(\Delta X, Z+\Delta Z)}{\Delta X}= \begin{cases}\frac{\sigma_{z}(\Delta X, Z+\Delta Z)-\sigma_{z}(\Delta X, Z)}{\Delta Z}, & \text { if } z+\Delta z=H(0), \\ \frac{\sigma_{z}(\Delta X, Z+2 \Delta Z)-\sigma_{z}(\Delta X, Z)}{2 \Delta Z}, & \text { if } z+\Delta z<H(0),\end{cases}
$$

or, after re-arrangement,

$$
\sigma_{z}(\Delta X, Z)= \begin{cases}\sigma_{z}(\Delta X, Z+\Delta Z)-\Delta Z\left[1-\varepsilon \frac{\tau(\Delta X, Z+\Delta Z)}{\Delta X}\right], & \text { if } z+\Delta z=H(0), \\ \sigma_{z}(\Delta X, Z+2 \Delta Z)-2 \Delta Z\left[1-\varepsilon \frac{\tau(\Delta X, Z+\Delta Z)}{\Delta X}\right], & \text { if } z+\Delta z<H(0) .\end{cases}
$$

Note that Equation (29a) is only a first-order derivative approximation, but after multiplying it by $\Delta Z$ and re-arranging the terms in order to obtain relation (30), we finally obtain a second-order approximation of $\sigma_{\mathrm{z}}(\Delta X, Z)$. Relation (29b) is itself a second-order derivative approximation, and so Equation (30b) gives a third-order approximation for $\sigma_{\mathrm{Z}}(\Delta X, Z)$.

Next, since $\partial \tau / \partial X$ is even, one has up to second order

$$
\partial \tau(0, Z) / \partial X=\partial \tau(\Delta X, Z) / \partial X .
$$

Consequently, Equation (30) also holds at $X=0$, so that

$$
\sigma_{z}(0, Z)= \begin{cases}\sigma_{z}(0, Z+\Delta Z)-\Delta Z\left[1-\varepsilon \frac{\tau(\Delta X, Z+\Delta Z)}{\Delta X}\right], & \text { if } z+\Delta z=H(0), \\ \sigma_{z}(0, Z+2 \Delta Z)-2 \Delta Z\left[1-\varepsilon \frac{\tau(\Delta X, Z+\Delta Z)}{\Delta X}\right], & \text { if } z+\Delta z<H(0) .\end{cases}
$$

With this we deduce, since $\Delta(0, Z)$ is already known,

$$
\sigma_{x}(0, Z)=\Delta(0, Z)+\sigma_{z}(0, Z) .
$$

Finally, function $\tau(\Delta X, Z)$ can be updated as follows: in Equation (7a), $\delta \sigma_{\mathrm{x}}$ and $\partial \tau / \partial Z$ at $(\Delta X, Z+\Delta Z)$ can be approximated. Then, as for relations (30a and b), we obtain

$$
\tau(\Delta X, Z)=\left\{\begin{array}{l}
\tau(\Delta X, Z+\Delta Z)+\frac{2 \varepsilon \Delta Z}{\Delta X}\left[\sigma_{x}(\Delta X, Z+\Delta Z)-\sigma_{x}(0, Z+\Delta Z)\right], \text { if } z+\Delta z=H(0), \\
\tau(\Delta X, Z+2 \Delta Z)+\frac{4 \varepsilon \Delta Z}{\Delta X}\left[\sigma_{x}(\Delta X, Z+\Delta Z)-\sigma_{x}(0, Z+\Delta Z)\right], \text { if } z+\Delta z<H(0) .
\end{array}\right.
$$

Thus, all functions at level $Z$ are determined, since

$$
\tau_{\mathrm{II}}(\Delta X, Z)=\frac{1}{4}\left[\sigma_{x}(\Delta X, Z)-\sigma_{Z}(\Delta X, Z)\right]^{2}+\tau^{2}(\Delta X, Z) .
$$

By this marching process, all variables at the divide and in the column just next to the divide are computed.

The procedure described above is based on a prescribed form of function $W(Z)$ of the divide, which was given by Equation (16). Note that by starting from any feasible function $W(Z)$, the above process can be repeated easily. For any function $W(Z)$, the quality of the fit of the resulting solution functions can be measured by adding the squares of the relative errors in satisfying Equations (7) and boundary conditions (9) and (10) at $X=0$ and $X=\Delta X$. Then, the corrrect choice will be that function $W(Z)$ which minimizes this overall added squared error. We refrain from presenting any details and pass directly to the presentation of numerical results.

\section{NUMERICAL EXAMPLE}

The procedure described in sections $3.1-3.3$ was tested in a hypothetical numerical example. The actual values of the parameters were selected as

$$
\begin{aligned}
H_{\mathrm{D}} & =0.8, \quad \alpha=0.2, \quad \beta=0.031, \quad v=0.002776, \\
s^{2} & =0.000308, \quad \varepsilon=(v)^{\frac{1}{2}} .
\end{aligned}
$$

The step sizes in both directions, $X$ and $Z$ were selected as

$$
\Delta X=\Delta Z=0.05 \text {. }
$$

In order to obtain a feeling of how the numerical results depend on the values of the above parameters, we systematically changed the value of one of the above parameters to obtain six cases, which were as follows:

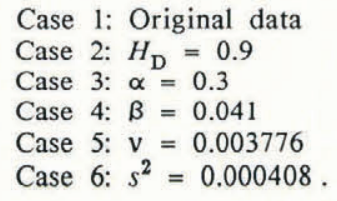

The temperature distributions for these cases are tabulated in Table III, and a typical shape (for original data) of function $T(0, Z)$ is drawn in Figure 2. For the 
TABLE III. TEMPERATURE DISTRIBUTION ON THE DIVIDE (VALUES IN CENTIGRADE ARE OBTAINED BY MULTIPLYING THE NUMBERS BY A FACTOR OF 20)

$T(0, Z)$

$\begin{array}{lllllll}Z & \text { Case } 1 & \text { Case } 2 & \text { Case } 3 & \text { Case } 4 & \text { Case } 5 & \text { Case 6 } \\ 0.0 & -0.204 & -0.237 & -0.197 & -0.165 & -0.202 & -0.203 \\ 0.05 & -0.254 & -0.287 & -0.247 & -0.215 & -0.252 & -0.253 \\ 0.1 & -0.304 & -0.337 & -0.297 & -0.265 & -0.303 & -0.304 \\ 0.15 & -0.349 & -0.383 & -0.343 & -0.311 & -0.348 & -0.349 \\ 0.2 & -0.388 & -0.423 & -0.382 & -0.352 & -0.387 & -0.387 \\ 0.25 & -0.419 & -0.456 & -0.414 & -0.388 & -0.418 & -0.419 \\ 0.3 & -0.444 & -0.482 & -0.439 & -0.417 & -0.443 & -0.443 \\ 0.35 & -0.462 & -0.502 & -0.457 & -0.440 & -0.461 & -0.461 \\ 0.4 & -0.474 & -0.517 & -0.470 & -0.458 & -0.474 & -0.474 \\ 0.45 & -0.483 & -0.528 & -0.480 & -0.471 & -0.483 & -0.483 \\ 0.5 & -0.489 & -0.535 & -0.486 & -0.480 & -0.488 & -0.489 \\ 0.55 & -0.492 & -0.540 & -0.490 & -0.487 & -0.492 & -0.492 \\ 0.6 & -0.495 & -0.543 & -0.493 & -0.492 & -0.495 & -0.495 \\ 0.65 & -0.497 & -0.545 & -0.496 & -0.495 & -0.497 & -0.497 \\ 0.7 & -0.498 & -0.547 & -0.497 & -0.497 & -0.498 & -0.498 \\ 0.75 & -0.499 & -0.548 & -0.499 & -0.499 & -0.499 & -0.499 \\ 0.8 & -0.500 & -0.549 & -0.500 & -0.500 & -0.500 & -0.500\end{array}$

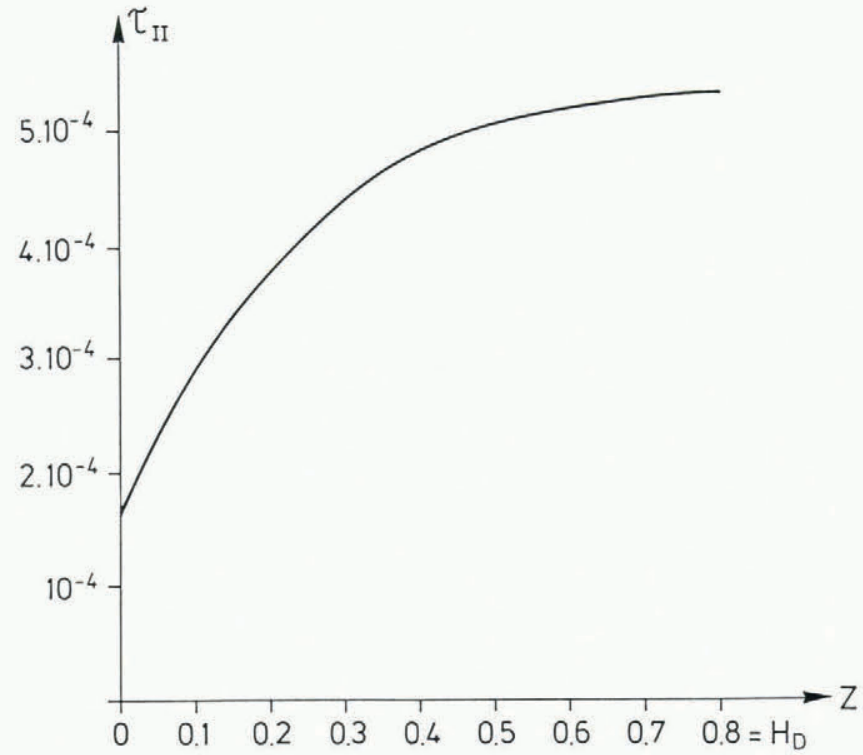

Fig. 4. Vertical distribution of the second stress-deviator invariant at the divide. (In the reduced model $\tau_{\text {II }}$ would vanish.)

Dordrecht, etc., D. Reidel Publishing Company/Tokyo, Terra Scientific Publishing Company.

Hutter, K. and L. Vulliet. 1985. Gravity-driven slow creeping flow of a thermoviscous body at elevated temperatures. J. Therm. Stresses, 8(1), 99-138.

Hutter, K., S. Yakowitz, and F. Szidarovszky. 1986. A numerical study of plane ice-sheet flow. J. Glaciol., 92(111), 139-160.

Hutter, K., S. Yakowitz, and F. Szidarovszky. 1987. Coupled thermomechanical response of an axisymmetric cold ice sheet. Water Resour. Res., 23(7), 1327-1339.

Johnson, R.E. and R.M. McMeeking. 1984. The surface flow in glaciers obeying Glen's law. Q. J. Mech. Appl. Math., 37, 273-291.

Morland, L.W. 1984. Thermomechanical balances of icesheet flow. Geophys. Astrophys. Fluid Dyn., 29, 237-266.

Raymond, C.F. 1983. Deformation in the vicinity of ice divides. J. Glaciol., 29(103), 357-373.

Yakowitz, S. and F. Szidarovszky. 1986. An introduction to numerical computation. New York, Macmillan Publishing Company.

Yakowitz, S., K. Hutter, and F. Szidarovszky. 1986. Elements of a computational theory for glaciers. $J$. Comput. Phys., 66(1), 132-150. 